\title{
Mitral valve surgery for functional mitral regurgitation: Should moderate to severe tricuspid regurgitation be treated? A propensity score analysis
}

Andrew S. Wechsler, MD

Any study designed to examine tricuspid valve intervention in patients with functional tricuspid valve regurgitation (FTR) associated with functional mitral valve regurgitation (FMR) begins with the understanding that FMR in this setting is different from FMR associated with structural mitral valve disease. Operations for structural mitral valve disease are anticipated to correct most of the abnormal physiologic characteristics associated with the volume overload of mitral regurgitation and to promote progressive and durable reverse remodeling. It is thus reasonable to intuit that mild degrees of FTR in this setting are likely to regress or remain the same as pulmonary artery pressures, right ventricular dimensions and function, and left ventricular properties improve with time.

Such is not the case for FMR associated with abnormal left ventricular systolic function. FMR can be surgically corrected with low operative mortality. However, there remains a high recurrence rate, particularly when severe FMR is treated by isolated valve repair rather than replacement. ${ }^{1}$ The early changes of reverse left ventricular remodeling are not necessarily sustained, and the progressive nature of the intrinsic cardiomyopathy (ischemic or dilated) commonly resumes after an initial period of improvement. ${ }^{2,3} \mathrm{~A}$ study from the Mayo Clinic found a clear correlation between the extent of functional mitral regurgitation and late mortality. Wu and colleagues, ${ }^{4}$ however, reported no benefit in longevity for patients undergoing operation for FMR in association with either ischemic or dilated cardiomyopathy. Pending further understanding through additional studies, correction of FMR may be thought of as setting back the adverse physiologic clock for a while but not stopping it from running.

Calafiore and colleagues ${ }^{5}$ used propensity matching to study a group of patients with FTR secondary to FMR. Almost all patients who had severe FTR underwent repair with a DeVega annuloplasty. Patients with mild to moderate FMR who did not have repair had decreased longevity and poorer survival in a low New York Heart Association functional class (ie, closer to class I). It was particularly interesting that patients with uncorrected mild to moderate FTR in

\footnotetext{
From the Drexel University College of Medicine, Philadelphia, Pa.

Received for publication June 10, 2008; accepted for publication July 10, 2008.

Address for reprints: Andrew S. Wechsler, MD, Drexel University College of

Medicine, 245 N. 15th St, MS 111, Philadelphia, PA 19102 (E-mail: andrew.

wechsler@drexelmed.edu).

J Thorac Cardiovasc Surg 2009;137:267-8

$0022-5223 / \$ 36.00$

Copyright (c) 2009 by The American Association for Thoracic Surgery

doi: $10.1016 / j$.jtcvs.2008.07.019
}

this population fared less well than patients with surgically corrected severe FTR. It is tempting to assume that impaired long-term outcome was related to recurrence of FMR, but a small study by Matsunaga and Duran ${ }^{6}$ dissociated diminished prognosis and return of FTR from return of FMR. The intimation is that, after repair of FMR, progression of intrinsic left ventricular chamber disease is a more important predictor of long-term outcome than is return of mitral regurgitation. This hypothesis could be tested by serially studying a cohort of patients who underwent chordal-sparing mitral valve replacement rather than repair for FMR.

Propensity matches balance populations for variables that are measured and included in the match. They do not balance for variables not included that may contain important information. In the cohort of patients studied by Calafiore and colleagues, ${ }^{5}$ there was great similarity among the patients, such that 100 of 110 patients were matched for the variables selected. The data are presented by degree of FTR before, early, and late after operation. Surgeons who treat patients with FTR require additional information to help individualize their treatment choices and to further enhance understanding of the disease processes. Specifically, a surgeon should know which variables predict mortality in the patients who underwent operation and the patients who experienced recurrent FTR. This information is particularly important for the variables that were not matched, such as details of right ventricular performance and chamber dimension beyond the tricuspid annular plane systolic excursion, which was measured as a rough indicator of right ventricular function.

Because multiple descriptors of left ventricular performance were included in the propensity match, one might hypothesize that the critical information determining longterm functional class and survival were related to properties of the right ventricle that were not described. This concept was indirectly substantiated in a recent study by de Bonis and colleagues. ${ }^{7}$ In their study, patients with tricuspid regurgitation grades less than $3+$ generally did not have corrected at the time of operation for FMR. During a 2-year interval, conditions in approximately $18 \%$ of these patients progressed 2 grades. Preoperative right ventricular function was shown to be an important predictor of worsening tricuspid regurgitation, but no attempt at correlation to late mortality was made. ${ }^{5}$ However, a study by Nath and colleagues, ${ }^{8}$ which corrected for differences in ventricular function and pulmonary artery pressure, demonstrated that worsening degrees of tricuspid regurgitation were associated with decreased longevity. 


\section{Abbreviations and Acronyms}

FMR $=$ functional mitral valve regurgitation

FTR $=$ functional tricuspid valve regurgitation

Propensity matching is a powerful tool that can create "matched" subjects, a logit that defines closely related subjects for the variables proposed or a number that can be used in a multivariable analysis. The technique is most effective in large populations when many variables are studied because it yields greater confidence that the patients included are similar and differ only in the particular variable of interest. In smaller studies with more similar populations, it may have been of interest to use traditional multivariable logistic regression analysis on the entire population to determine whether other variables might have had an important effect. This is particularly true for the interaction between ischemic cardiomyopathy and the lesser likelihood of tricuspid valve repair in that population and the potential for a different long-term outcome between ischemic and idiopathic cardiomyopathy, independent from the tricuspid valve repair. One would like to be certain that it was failure to address the moderate FTR that was the critical factor in survival rather than the underlying condition that created the FMR.

A potential confounding factor in the study by Calafiore and colleagues ${ }^{5}$ may relate to quantification of tricuspid regurgitation. The extent of tricuspid regurgitation seen on the echocardiogram is variable over time and heavily dependent on the loading condition of both ventricles when the studies are performed. The inclusion of patients designated as having mild to moderate FTR in the unrepaired group who may have had moderate or severe FTR could have had some effect on the outcome data. Standardization of the extent of tricuspid regurgitation is extremely difficult, so much so that
Dreyfus and colleagues ${ }^{9}$ reported a series of patients in whom the decision to repair or not repair the tricuspid valve was made on an anatomic basis (annular dilation) rather than a functional basis.

This study is an important addition to our information base about FMR in the setting of ischemic mitral regurgitation. As Calafiore and colleagues ${ }^{5}$ make clear, the decision to perform a tricuspid procedure when performing a mitral procedure is not terribly difficult. Tricuspid repairs can be performed with minimal to no additional myocardial ischemic time, are inherently simple, and have little risk of additional operative morbidity while potentially conferring long-term benefit, as demonstrated in this report.

\section{References}

1. McCarthy PM, Budhia SK, Raheswaran J, Hoerscher KJ, Lytle BW, Cosgrove DM, et al. Tricuspid valve repair: durability and risk factors for failure. J Thorac Cardiovasc Surg. 2004;127:674-85.

2. Hung J, Papakostas L, Tahta SA, Hardy BG, Bollen BA, Duran CM, et al. Mechanism of recurrent ischemic mitral regurgitation after annuloplasty: continued LV remodeling as a moving target. Circulation. 2004;110(11 Suppl. 1):II8.

3. Patel JR, Borgeson DD, Barnes ME, Rihal CS, Daly RC, Redfield MM. Mitral regurgitation in patients with advanced systolic heart failure. J Card Fail. 2004;10: 285-91.

4. Wu AH, Aaronson KD, Bolling SF, Pagani FD, Welch K, Koelling TM. Impact of mitral valve annuloplasty on mortality risk in patients with mitral regurgitation and left ventricular systolic dysfunction. J Am Coll Cardiol. 2005;45:381.

5. Calafiore MA, Gallina S, Iacò AL, Contini M, Bivona A, Gagliardi M, et al. Mitral valve surgery for functional mitral regurgitation. Should moderate-or-more tricuspid regurgitation be treated? A propensity score analysis. J Thorac Cardiovasc Surg. 2008; 137:267-9.

6. Matsunaga A, Duran CMG. Progression of tricuspid regurgitation after repaired functional ischemic mitral regurgitation. Circulation. 2005;112(9 Suppl):I453-7.

7. De Bonis M, Lapenna E, Sorrentino F, La Canna G, Grimaldi A, Maisano F, et al. Evolution of tricuspid regurgitation after mitral valve repair for functional mitral regurgitation in dilated cardiomyopathy. Eur J Cardiothorac Surg. 2008; 33:600-6.

8. Nath J, Foster E, Heidenreich PA. Impact of tricuspid regurgitation on long-term survival. J Am Coll Cardiol. 2004;43:405-9.

9. Dreyfus GD, Corbi PJ, Chan KM, Bahrami T. Secondary tricuspid regurgitation or dilatation: which should be the criteria for surgical repair? Ann Thorac Surg. 2005; $79: 127-32$ 